\title{
PTSD and severe panic disorder post total gastrectomy: a case of fast response to the psychopharmacology therapy associated with reinsertion of spiritual practices
}

\begin{abstract}
This case report describes a 28 year-old female patient that, after being submitted to total gastrectomy, retroperitoneal lymphadenectomy and splenectomy due to a gastric leiomyosarcoma followed by several interventions as a consequence of complications, developed post traumatic stress disorder (PTSD) and frequent severe panic attacks. Fluoxetine, quetiapine and periciazine were administered as treatment choice via jejunostomy. The involvement of the patient's family associated to a reinforcement in spiritual practices enhanced the therapeutic response, bringing the patient back to an emotional equilibrium after 4 days since treatment onset, becoming asymptomatic regarding the anxiety symptoms, allowing her to return to her social life.
\end{abstract}

Keywords: total gastrectomy, panic disorder, ptsd, palliative care
Volume 2 Issue 3 - 2018

\author{
Pierre FS, ' Silva CM, ${ }^{2}$ de Avellar ML, ${ }^{3}$ \\ Medeiros SK, ${ }^{4}$ Silva RV, ${ }^{5}$ Coronato-Nunes T ${ }^{6}$ \\ 'Family and Community Medicine Specialist, Brazil \\ 2Medical Student, Faculdade de Medicina de Petrópolis, Brazil \\ ${ }^{3}$ Infectious Diseases Student, University of Edinburgh, United \\ Kingdom \\ ${ }^{4}$ Clinical Radiologist at Instituto Nacional do Câncer (INCA), \\ Brazil \\ ${ }^{5}$ General and Oncological Surgeon, Teacher on General Surgery, \\ Faculdade de Medicina de Petrópolis, Brazil \\ ${ }^{6}$ Psychiatrist, MSc Biomedical Sciences, Teacher on Psychiatry, \\ Faculdade de Medicina de Petrópolis, Brazil
}

\begin{abstract}
Correspondence: Fabiana Santos Pierre, Rua, Family and Community Medicine Specialist, Alencar Lima 35, room 614, Centro, Petrópolis-RJ, CEP-25620-050, Brazil, Email fabiana_pierre@hotmail.com
\end{abstract}

Received: April 26, 20I8 | Published: June 21, 2018

\section{Case report}

The patient described was a 28 year-old female, single, catholic, and teacher. The patient presented dry cough, extreme exhaustion and severe anaemia and had an upper gastrointestinal endoscopy performed to investigate the case, revealing an extensive lesion at the gastric fundus. The patient was subjected to total gastrectomy and Roux-en-Y esophagojejunostomy, removing adjacent lymph nodes, splenectomy and jejunostomy for nutrition (Figure 1). The pathological diagnosis was leiomyosarcoma. Postoperatively, the patient developed afferent loop syndrome, submitted to laparotomy, where was identified wound dehiscence on duodenal stump, esophageal anastomosis and jejunostomy, developing peritonitis and severe sepsis. The patient was submitted to several surgery procedures, presenting other complications such as perforation of gallbladder and small intestines caused by septic embolism, originating an enteric fistula. She remained for 68days in Intensive Care Unit and another 47days in an infirmary private room. During hospitalization, the patient started developing anxiety symptoms, being prescribed Escitalopram 10mg, drops via jejunostomy. Haloperidol and Promethazine via intramuscular were administered in case of anxiety crisis.
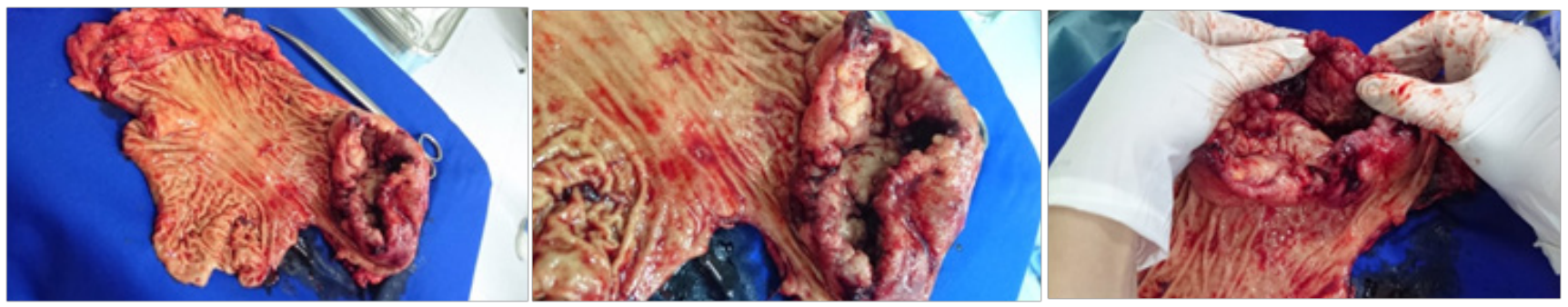

Figures I Leiomyosarcoma em gastric fundus ( $17 \mathrm{~cm}$ in diameter)

Patient was discharged with esophagostomy, jejunostomy (for nutrition) and enteric fistula in peritoneostomy wound. After two weeks being asymptomatic, the patient developed esophageal stenosis, requiring endoscopic balloon dilation. At home, the patient was under intensive home care. She presented exacerbation of anxiety, also presenting panic attacks episodes, contorting her body on bed, screaming for help, feeling fear and sense of impending doom, besides mental confusion, emotional suffering, constant irritability and insomnia. Also developed the idea of not tolerating new visits to the hospital for surgical procedures. Escitalopram doses were increased to $15 \mathrm{mg}$ and initiated Clonazepam $2 \mathrm{mg}$ at night, but no improvement on the symptoms was observed on the following days. At this point, 
the patient presented 10panic attacks per day, screaming and moaning during all the period she was awake. On the first domiciliary visit of our health care team, the different dimensions of human suffering were addressed, prioritizing the spiritual, emotional and familiar aspects. During the interview, it was possible to identify that the family was being highly affected by the gradual emotional decline of the patient, which needed a focused mental and emotional support due to her suffering, besides reevaluating the drugs scheme which did not present any improvement on the symptoms.

Macerated and diluted Quetiapine pills for jejunostomy administration, as $25 \mathrm{mg}$ in the morning, $25 \mathrm{mg}$ in the afternoon and $100 \mathrm{mg}$ at night. Escitalopram was gradually removed and Fluoxetine $20 \mathrm{mg} / \mathrm{mL}$ started as 5 drops each 3 days, until $30 \mathrm{mg}$ per day via jejunostomy. Periciazine $40 \mathrm{mg} / \mathrm{mL}$ was used in case of anxiety crisis, as 10 drops via jejunostomy, as many times as necessary, until a maximum of 10 times per day. On the first day with the new drug scheme, the patient presented hypotension, limiting her daily activities, but presented a significant improvement on her sleep pattern and decreased the number and intensity of anxiety crisis, presenting 4 panic attacks in 24hours (using Pericyazine 4times to revert it). On the following day, Quetiapine was reduced to $25 \mathrm{mg}$ in the morning and $50 \mathrm{mg}$ at night. She presented 6 anxiety crisis, with Pericyazine use in all 6 to revert the symptoms. On the third day of new drug scheme, a spiritual approach was added to her therapy, since then she did not present any anxiety crisis, annulating the need of emergencial use of Pericyazine. Fluoxetine $30 \mathrm{mg}$ and quetiapine $25 \mathrm{mg}$ in the morning and $50 \mathrm{mg}$ at night were maintained as her treatment. Blood pressure remained stabilized, started to increase her appetite, decreased the nausea and presented a desire to interact in social activities.

\section{Discussion}

Approximately half of the patients with advanced cancer meet criteria for psychiatric disorders. ${ }^{1}$ Although it is not being described the case of patients developing post-traumatic stress disorder (PTSD) after total gastrectomy, there are various reports of patients developing this disorder after extensive surgeries to remove cancer in many different organs. ${ }^{2,3}$ The patient in this case, after being diagnosed with cancer, was submitted to successive surgeries, a long period of hospitalization and returned home with several physical limitations. The PTSD diagnosis is based on the disproportional fear to return to the hospital, reviving the hospitalization and surgical experiences in an intrusive form, both awaken and in her dreams, developing many panic attacks during the day. The drug administration was performed on the jejunostomy because there was partial lost on the esophagostomy when ingested orally. The chosen medication had to be in solution or macerated and diluted to avoid clogging the probe.

There are many possible therapeutic schemes for PTSD and panic disorder treatment. ${ }^{4-6}$ A selective serotonin reuptake inhibitor is normally an option for the treatment, however, in case of intense anxiety and panic crisis, it is necessary to associate other classes of drugs to bring rapid relief. Exchanging Escitalopram for Fluoxetine was based on the idea of using a drug with a longer therapeutical interval in case of refractory symptoms. Clonazepam use at night was unsuccessful, despite being a good medication to revert anxiety crisis. Use of the antipsychotic Quetiapine, which is not available in solution, had to be carefully analysed, choosing the most soluble pill to dilute. As it presents hypotension as a side affect, the doses had to be adjusted afterwards. The first generation antipsychotic Pericyazine was a good choice as it was available in solution and presents a safe therapeutic interval (until $300 \mathrm{mg} /$ day), with fast anxiolytic action. The dose has to be individually analysed in each case, considering that higher doses have a higher sedative effect.

In all the domiciliary visits, the aspects of spiritual suffering were accessed and traditional religious family practices were encouraged. On the third day of new treatment, the patient showed desire to receive the Eucharist in her house (an important ritual in Western Catholic Church). After receiving the Eucharist and maintaining the drug scheme, the patient developed emotional stability, ceasing completely the crisis and was reinserted into her social and familial circle. It is well established that religious practices lead to an increase on the belief of recovering, enhance motivation, diminishes anxiety and augment the capacity of facing the uncertain situations that are about to happen..$^{7-9}$

\section{Conclusion}

The peculiarity of this case rely on the fact of the rapid response of the patient with severe anxiety crisis in less than 4days. This happened when properly adjusting the drugs, introducing medication that have a fast response in controlling the crisis and adequate doses. However, it is also important to emphasize the welcoming involvement of the family, the affectionate interaction of our team with the patient and the reintroduction of faith and spiritual practices for all the people involved presented an important healing effect, considering the fast improvement on her health, which was not observed with any previous intervention. To conclude, it is relevant to point that even the scientific intervention had a therapeutic effect. When soliciting to the patient and her family to authorize her data to be used to produce this article, she revealed us that the publication of this article brought her a deep meaning for her illness. She told us, thrilled and with tears in her eyes "It is like I can now understand the reason of all this. Maybe it happened so it can help other people like me. It will be an honour to see my story helping other people."

\section{Acknowledgements}

None.

\section{Conflicts of interests}

The author declares no conflict of interest.

\section{Patient consent form}

The patient consent the use of the data collected to be published by signing a Patient Consent Form.

\section{References}

1. Miovic M, Block S. Psychiatric disorders in advanced cancer. Cancer. 2007;110(8):1665-1676.

2. Chan $\mathrm{CMH}, \mathrm{Ng} \mathrm{CG}$, Taib NA, et al. Course and predictors of posttraumatic stress disorder in a cohort of psychologically distressed patients with cancer: A 4-year follow-up study. Cancer. 2018;124(2):406-416.

3. Jeantieu M, Gaillat F, Antonini F, et al. Postoperative pain and subsequent PTSD-related symptoms in patients undergoing lung resection for suspected cancer. J Thorac Oncol. 2014;9(3):362-369.

4. Koirala R, Søegaard EGI, Thapa SB. Updates on Pharmacological Treatment of Post-Traumatic Stress Disorder. JNMA J Nepal Med Assoc. 2017;56(206):274-280. 
5. Xu JJ, Chan MJ, Yang YC. Fluoxetine as a treatment for post-traumatic stress disorder. Neurosciences. 2011;16(3):257-262.

6. Jeffreys M, Capehart B, Friedman MJ. Pharmacotherapy for posttraumatic stress disorder: review with clinical applications. J Rehabil Res Dev. 2012;49(5):703-715
7. Tsai TJ, Chung UL, Chang CJ, et al. Influence of Religious Beliefs on the Health of Cancer Patients. Asian Pac J Cancer Prev. 2016;17(4):23152320 .

8. Torabi F, Rassouli M, Nourian M, et al. The Effect of Spiritual Care on Adolescents Coping With Cancer. Holist Nurs Pract. 2018;32(3):149159. 